\title{
蚱蝉听觉中间神经元对声信号时间 参数的编码*
}

\author{
沈钧贤 \\ （中国科学院生物物理研究所,北京 100101）
}

\section{关链词听觉中间神经元、时间参数、声信息编码、蚱蝉}

动物鸣声的时域信息,通常是种属专一的,表达一定的“语意”,对进行种内或种间声通信 的动物来说具有十分重要的意义 ${ }^{[1,2]}$. 在探讨声模式识别的机理时, 有人假设动物听觉通路中 有专门的时间速率识别器 (rate recognizer), 或时间调谐神经元 (temporally tuned neurons), 但至今没有实验证据; 有的认为听中枢内存在某种速率带通 (rate band-pass) 细胞, 在蛙脑中 已经找到 ${ }^{[3]}$.

蝉是昆虫中利用声音进行种内通信的代表. 蝉鸣声的主要声学特征基本上固定不变, 并 且是种属专一的. 雌蝉主要依据鸣声的时域信息来辨认同种雄蝉发出的鸣声, 然后趋声飞向 雄蝉实现配对. 文献 [4]的实验结果表明, 虾蝉的初级听觉神经元具有时锁 (time-locking) 功 能,基本上能以同步码方式“复制”鸣声的多种时间参数.

本文将报道用神经电生理方法在单细胞水平上研究蚱蝉听觉中间神经元对声信号时间参 数的编码、加工与传递特征, 为阐明动物听觉模式识别的机理提供新的实验资料.

\section{1 材 料 与 方 法}

\section{1 材料}

成年雄与雌性蚱蝉 Cryptotympana atrata (同翅目,蝉科) 28 只在夏季采集于北京西郊的 树林中,按性别分开饲养在昆虫箱内, 室温约 $25^{\circ} \mathrm{C}$.

\section{2 标本制备}

剪去蝉的双翅和胸肢,用粘合剂将其背部粘固在金属小架上,腹面朝上. 暴露前胸神经 节. 用注有 $3 \mathrm{molKCl}$ 溶液的玻璃微电极 (阻抗 $20 \mathrm{M} \Omega$ 以上) 从前胸神经节记录听觉神经元的 声反应活动, 并逐项测定该神经元的有关生理特征. 用日本光电 DAB-1100 型直方图机从听 觉细胞对每个刺激组的 50 次重复的反应得到刺激后时间直方图 (poststimulus time histogram, PSTH). 每个计算贮存 (bin) 时宽为 $0.5 \mathrm{~ms}$. 有些数据由美国 DATA6000 型波形分 析仪采样,采样时间 $20 \mu \mathrm{s}$, 储存在软盘中,备以后分析.

\section{3 声刺激}

本文所用的单脉冲声刺激为短纯音, 上升、下降时间各为 $1 \mathrm{~ms}$, 脉冲时程 (pulse duration, PD)变动范围为 $2-100 \mathrm{~ms}$. 所用的多脉冲信号含脉冲数 6 个,每个脉冲的 PD 为 6 或 $2 \mathrm{~ms}$, 上

1993-05-05 收稿, 1993-08-17 收修改稿.

- 国家自然科学基金资助项目. 
升下降时间为 1 或 $0.1 \mathrm{~ms}$,脉冲重复间隔 (pulse repetition interval, PRI) 为 PD 的 2 倍. 以上 参数包含了蝉鸣声的典型时间信息. 由刺激程序发生器 (英国 HG-100 型.) 输出电信号,经功 率放大后驱动宽带扬声器产生声脉冲. 声脉冲的载频选用虾蝉鸣声的主频率 $4.5 \mathrm{kHz}$. 声强以 $\mathrm{dBSPL}$ (声压级) 表示, $0 \mathrm{~dB}$ 等于 $20 \mu \mathrm{Pa}$, 精度 $\pm 1 \mathrm{~dB}$. 提供声刺激的速率 1 次/s. 实验在电磁屏 蔽、隔声、防震的消声室内进行.

\section{2 结果与 讨 论}

业已证实在蚱蝉前胸神经节内有两类四种听觉中间神经元, 其声反应的生理特征详见文 献 $[5,6]$. 一类为紧张型 (tonic) 放电听觉细胞 (T1 和 T2), 它们在适宜声强和频率的声刺激下 有一串锋电位 (spike) 发放; 另一类为相位型 (phasic) 放电听觉细胞 (P1 和 P2), 它们对适宜声 刺激的反应是 $1-3$ 个锋电位发放, 放电时程很短.

\section{1 单脉冲信号时程的神经编码}

选择适宜的声刺激条件: 声强为阈上 $30 \mathrm{~dB}$, 声信号频率 $4.5 \mathrm{kHz}$, 单脉冲信号时程 (PD) 在 较大范围内 $(5-100 \mathrm{~ms})$ 变动. 这样的声刺激从两类听觉中间神经元所诱发的动作电位的放电 时程(firing duration, FD, 以首锋与末锋的时差表示)各有明显的特点: 紧张型听觉神经元的 放电时程能较好地跟随 PD 的变动 (图 1(a),(b)), 而相位型听神经元的 FD 基本上不变, 与声 刺激时程无关,例如 P1 的 FD 约为 $6 \mathrm{~ms}$ (图 1(c),(d)).

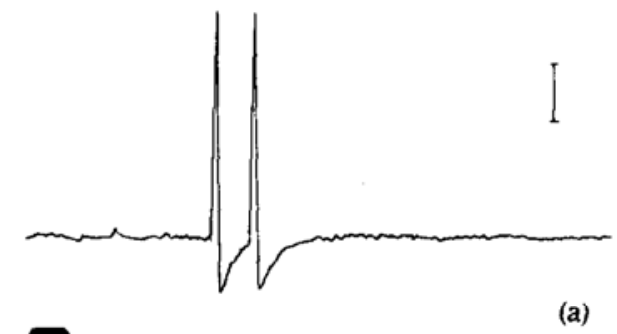

$5 \mathrm{~ms}, 4.5 \mathrm{kHz}$

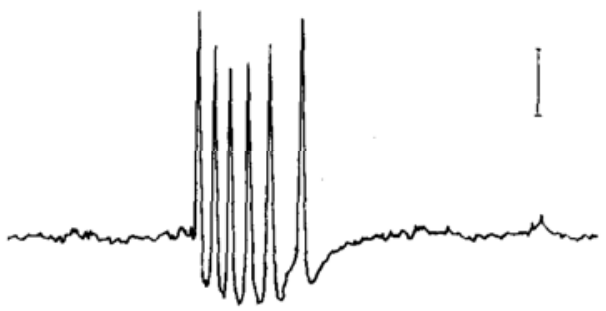

(b)

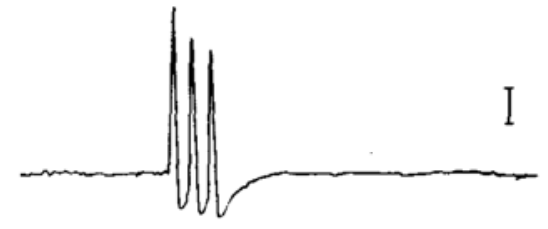

(c) $20 \mathrm{~ms}, 4.5 \mathrm{kHz}$

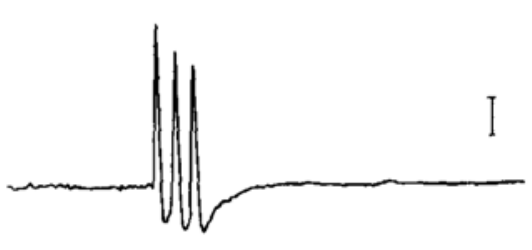

(d)

$20 \mathrm{~ms}, 4.5 \mathrm{kHz}$

$40 \mathrm{ms̀}, 4.5 \mathrm{kHz}$

图 1 蚱蝉紧张型听觉中间神经元 T2( (a), (b)) 和相位型听觉中间神经元 P1( (c), (d))对不同单脉冲信号 时程(PD)反应的示波图

每图的下线为声刺激: 声强为阈上 $30 \mathrm{~dB}$, 频率 $4.5 \mathrm{kHz}$; 竖线标尺: $10 \mathrm{mV}$

从听觉细胞对每个刺激组的 20 次重复的反应,计算两个反应量 : 即每次刺激所诱发的锋 
电位数 (spike count, SC) 的平均值和这些锋电位发放的持续时间 (FD) 的平均值. 将 SC 与 FD 以最大反应的百分比表示, 分别对 PD 作图,得到该神经元的时程反应函数. 如图 2 所示, 紧张型 (a) 与相位型 (b) 听觉神经元在对单脉冲信号时程编码的方式明显不同. 对紧张型听觉 神经元来说, SC 和 FD. 两条曲线具有长通 (long-pass) 滤波特征, 即 SC 和 FD 两者随着 PD 的 增加而增大, 相关系数接近 1. 而相位型听神经元则完全不同:SC 与 FD 两条曲线表现为全通 (all-pass)滤波特征,即其平均锋数和放电时程两者基本上与声刺激时程无关.
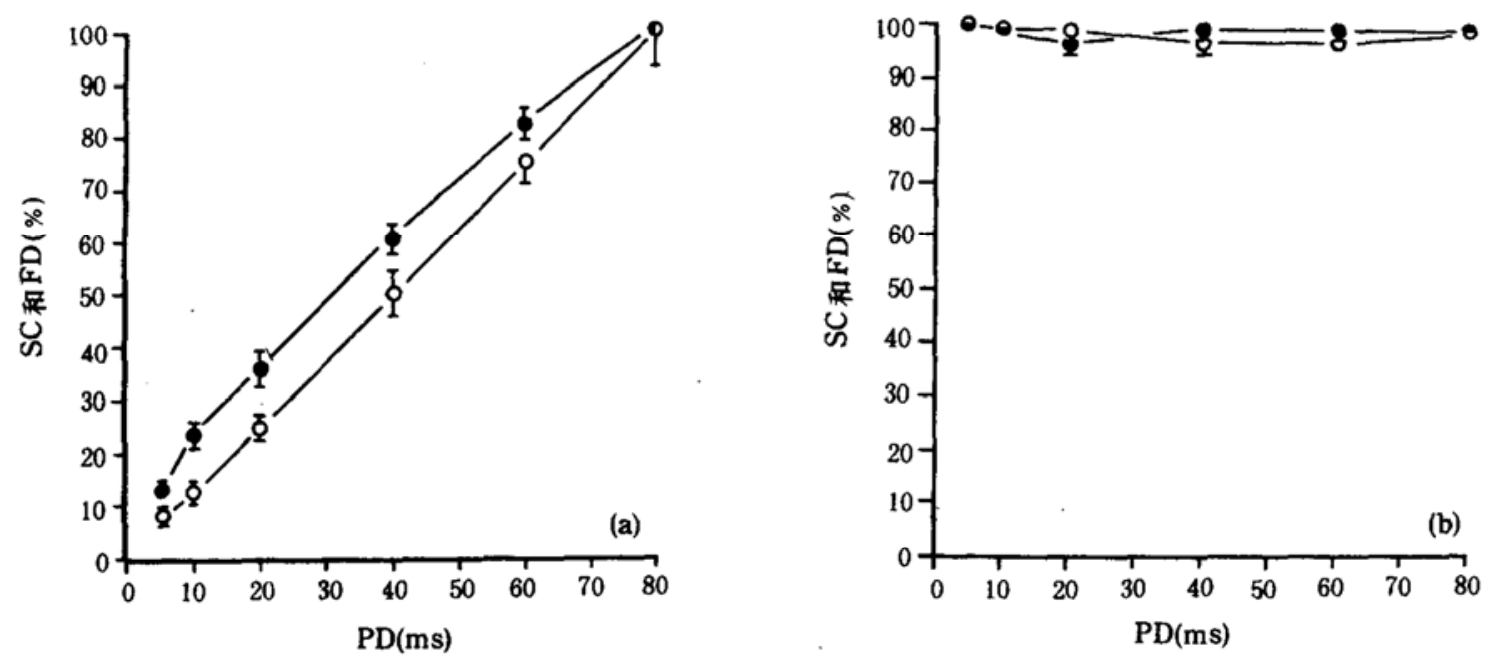

图 2

蛜蝉听觉中间神经元对单脉冲信号时程 PD 的反应函数, 分别以锋电位数 (SC, , 和锋电位发放的持续时间 (FD, O) 的 百分数表示. (a) 紧张型 $\mathrm{T} 2$ 神经元, (b) 相位型 P1 神经元. 实验为 20 次,垂直线为标准离差

\section{2 多脉冲信号时间参数的编码}

选择适宜的多脉冲信号作为声刺激, 声强为阈上 $30 \mathrm{~dB}$, 声信号频率等于蚱蝉鸣声的主频 率 $(4.5 \mathrm{kHz})$. 从听觉细胞对每个刺激组的 50 次重复的反应得到 PSTH, 它提供了该细胞对不 同声刺激反应的时间放电模式的详尽描述.

\section{2. 1 对 PD 为 $6 \mathrm{~ms}$, PRI 为 $12 \mathrm{~ms}$ 的六脉冲刺激的反应}

图 3.表示紧张型听觉神经元 (a) 和相位型听神经元 (b) 对 PD 为 $6 \mathrm{~ms}, \mathrm{PRI}$ 为 $12 \mathrm{~ms}$ 的六脉 冲信号反应的 PSTH. 从 PSTH 计算三个反应量: 即与每个脉冲相对应的锋数 (SC) 和放电时 程(FD)，以及锋发放的重复间隔 (firing repetition interval, FRI).

紧张型听觉神经元 (如 T2) 声反应的 SC 平均值依次为 $4,2,2,2,2,2$ 和 2 , 每组锋的 FD 平 均值依次为 $8.2,5.2,4.4,4.8,5.0$ 和 $5.2 \mathrm{~ms}$, 标准离差为 $\pm 0.2-0.6 \mathrm{~ms}$, 看来每个 FD 值不全 相同, 且与 PD $(6 \mathrm{~ms})$ 有一定差距; FRI 平均值分别为 $11.2,12.4,12.4,12.0$ 和 $12.0 \mathrm{~ms}$ 标准离 差范围较小 (小于 $\pm 0.4 \mathrm{~ms}$ ), 与 PRI $(12 \mathrm{~ms}$ ) 非常接近. 因此, 可以认为紧张型听觉中间神经元 能以 FRI 对声刺激的脉冲数和 PRI 编码.

·相位型听觉中间神经元 (如 P1) 的声反应 SC 分别为 2 或 $3,1,1,1,1$ 和 1 , 除第一个 FD 约 为 $6 \mathrm{~ms}$ 外, 其余的无 FD 可言, 而 FRI 大体上与 PRI 相等. 看来在高声强刺激条件下(如阈上 $30 \mathrm{~dB}), \mathrm{P} 1$ 能对脉冲数目和 PRI 编码. 另一个相位型神经元 P2 仅对多脉冲信号中的第一个, 或至多第二个, 有一个锋发放 (未表示在图上), 因此, 可以认为 P2 既不能对声脉冲数目, 也不 能对 PD 及 PRI 编码. 


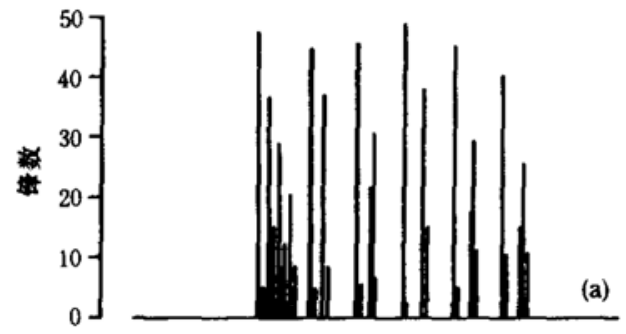

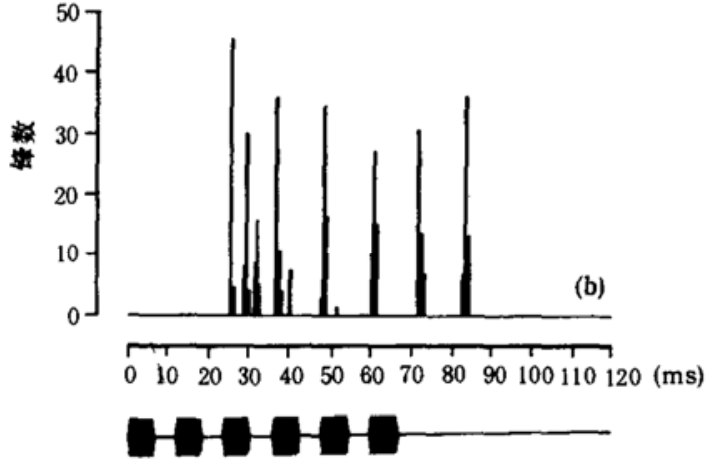

图 3 蚱蝉听觉中间神经元对六脉冲声信号 (PD 为 $6 \mathrm{~ms}$ )反应的 PSTH

Oms 表示刺激开始; 采样 50 次.（a)紧张型 $\mathrm{T} 2$ 神经元, (b) 相位型 P1 神经元

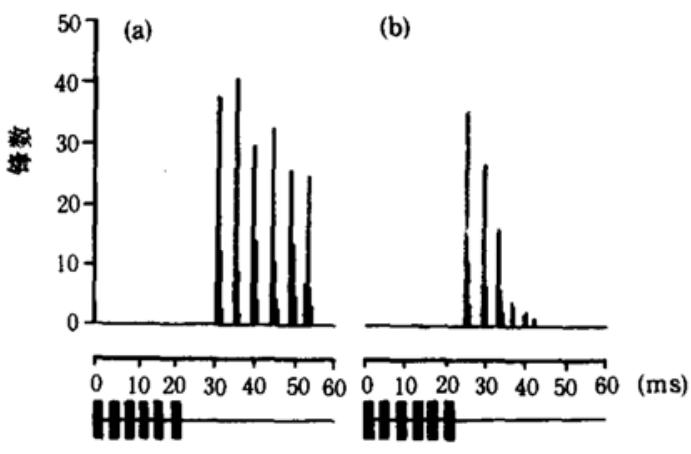

图 4 蜪蝉听觉中间神经元对六脉冲声信号 (PD 为 $2 \mathrm{~ms}$ ) 反应的 PSTH $0 \mathrm{~ms}$ 表示刺激开始; 采样 50 次. (a) 紧张型 T2 神 经元, (b) 相位型 P1 神经元

\section{2. 2 对 PD 为 $2 \mathrm{~ms}, P R I$ 为 $4 \mathrm{~ms}$ 的六脉冲刺激的反应}

当声刺激的 PD 为 $2 \mathrm{~ms}$, PRI 为 $4 \mathrm{~ms}$ 时, 与雄蚱蝉的自然鸣声非常接近 ${ }^{[5,6]}$, 紧张型听觉中 间神经元 (如 T2) 和相位型听觉中间神经元 (如 P1) 声反应的 PSTH 如图 4(a), (b) 所示. 对 $\mathrm{SC}, \mathrm{FD}$ 与 FRI 三个量的统计结果表明, 紧张型听觉神经仅基本上能以 SC 和 FRI 分别对声脉 冲数和 PRI 编码, 而无法得到 FD 值; 相位型听神经元仅对前二、三个声脉冲有单锋反应,FRI 仍与 PRI 相当, 也无 FD 值, 而对其余声脉冲基本上没有反应. 这里, FRI 约为 $4 \mathrm{~ms}$, 标准离差 小于 $\pm 0.4 \mathrm{~ms}$, 与从蚱蝉初级听觉神经元测出的最小时间分辦率是一致的 ${ }^{[4]}$.

从上述实验结果看来, PRI 和声脉冲数目能被蝉听觉系统中多种神经元编码, 可以认为它 们是蚱蝉声通信中重要的载信息时间参数,尽管我们尚未用自然鸣声直接进行类似实验. 另 外,脑中高一级听觉神经元的信息加工和模式识别特征还有待于深入研究.

\section{参考文献}

[1] Busnel. R. G. , Acoustic Behaviour of Animals, Elsevier, Amsterdam,1963.

[2] Elsner, N. , Popov, A. V. , Adv. Insect Physiol. , 1978,13:229-355.

[3] Capranica, R. R., Rose, G. , in Neuroethology and Behavioral Physiology, Springer, Berlin, Heidelberg, 1983,136 $-152$.

[4] 沈钧贤,科学通报, $1993,38(4): 363-366$.

[5] 沈钧贤等,声学学报, $1992,17(5): 382-386$.

[6] Shen Jun-xian, Chin. J. Acoust. , 1988,7(2):164-170. 